\title{
Aggregation of Partial T-Indistinguishability Operators
}

\author{
Tomasa Calvo Sánchez ${ }^{a}$ and Pilar Fuster-Parra ${ }^{b}$ \\ ${ }^{a}$ Department of Computer Sciences, Universidad de Alcalá, Alcalá de Henares, Madrid, Spain, \\ tomasa.calvo@uah.es \\ ${ }^{b}$ Department of Mathematics and Computer Sciences, Universitat Illes Balears, Palma de Mallorca, Baleares, Spain, \\ pilar.fuster@uib.es
}

\begin{abstract}
We focus on the aggregation of partial $T$ indistinguishability operators. We provide a characterization of those functions that allow to merge a collection of partial $T$ indistinguishability operators into a new one in terms of $(+$, max $)$-tuples. Moreover, we see that this is equivalent to the characterization by means of $\left(T, T_{M}\right)$-tuples given by Calvo et al. in [8]. Also, we analyze the aggregation of a collection $\left(E_{i}\right)_{i=1}^{n}$ of partial $T_{i^{-}}$ indistinguishability operators. Moreover, we show that a generalized inter-exchange composition function condition is a sufficient condition to guarantee that a function merges partial $T_{i}$-indistinguishability operators into a single one. Finally, we give different expressions of those aggregation functions defined by means of the additive generators of the corresponding t-norms and another specific function.
\end{abstract}

Keywords: Aggregation function, generalized inter-exchange composition function, partial indistinguishability operator, partial pseudo-metric, (+, max)-tuple, t-norm, tconorm, $\left(T, T_{M}\right)$-tuple, weighted function.

\section{Introduction}

Aggregation functions constitute an important tool in the field of information fusion (see $[4,5]$ ). The information can be given by means of fuzzy relations that depend on different applications (see [11, 18, 25, 26, $32])$. The problem of aggregating fuzzy relations has received considerable attention from the fuzzy community researching (see, [12, 20, 21, 27, 28, 29]), for this reason, in this work, we pay special attention in the preservation of the properties of partial $T$-indistinguishability operators by means of aggregation func- tions.

The early work of partial $T$-indistinguishability operators were introduced by Trillas in [30], so that given a t-norm $T:[0,1]^{2} \rightarrow[0,1]$, a $T$-indistinguishability operator on a (non-empty) set $X$ is a fuzzy relation $E: X \times X \rightarrow[0,1]$ verifying for all $x, y, z \in X$ the following properties: (i) $E(x, x)=1$, (ii) $E(x, y)=$ $E(y, x)$, (iii) $T(E(x, y), E(y, z)) \leq E(x, z)$. A $T$-indistinguishability operator is a mathematical tool for classifying objects when a measure presents some kind of uncertainty. They are also known as measures of similarity, in fact, the greater $E(x, y)$ the more similar are $x$ and $y$. Throughout this contribution we will assume that the reader is familiar with the basic notions of triangular norms, for more details see [17].

The $T$-indistinguishability operators are widely related to pseudo-metrics. In fact, one can find different methods, in the actual literature, to generate pseudometrics from indistinguishability operators, and viceversa (see $[1,2,15,19,21,24,29,31])$. According to [10], a pseudo-metric on a (non-empty) set $X$ is a function $d: X \times X \rightarrow[0, \infty]$ which satisfies the following axioms for all $x, y, z \in X$ : (i) $d(x, x)=0$, (ii) $d(x, y)=$ $d(y, x)$, (iii) $d(x, z) \leq d(x, y)+d(y, z)$. Pseudo-metrics are measures of dissimilarity, the smaller $d(x, y)$ the more similar are $x$ and $y$, and these measures are the antecedent of partial-pseudo-metrics.

The notion of partial pseudo-metric was introduced by Matthews (see [6]), he wanted to develop a suitable mathematical tool for quantitative models in denotational semantics. A partial pseudo-metric on a non-empty set $X$ was defined as a function $p$ : $X \times X \rightarrow[0, \infty[$ which satisfies for all $x, y, z$ the following: $(i) p(x, x) \leq p(x, y),(i i) p(x, y)=p(y, x)$, (iii) $p(x, y)+p(z, z) \leq p(x, z)+p(z, y)$. In the partial framework, a closely related notion of indistinguishability operator is considered in the works by Demirci [9] and by Bukatin et al. [6]. This new type of indistinguishability operator was called partial $T$-indistinguishability operator and was intro- 
duced in [14]. So that, we recall that a partial $T$ indistinguishability operator $E$ on a non-empty set $X$ is a fuzzy relation $E: X \times X \rightarrow[0,1]$ satisfying the following properties for any $x, y, z \in X$ : (i) $E(x, y) \leq E(x, x),($ ii $) E(x, y)=E(y, x)$, and (iii) $T(E(x, z), E(z, y)) \leq T(E(x, y), E(z, z))$. A partial $T$-indistinguishability operator $E$ on a non-empty set $X$ will be called a partial $T$-equality provided that it satisfies the following additional property for any $x, y, z \in X:\left(i^{\prime}\right) E(x, y)=E(x, x)=E(y, y)$ if and only if $x=y$, i.e., it is a partial $T$-indistinguishability operator on a set $X$ that separates points. In the literature, there are partial indistinguishability operators that are not an indistinguishability one, as for instance: $E_{k}(x, y)=k$, where $\left.k \in\right] 0,1\left[\right.$. Note that, $E_{k}$ is not a indistinguishability operator, since $E_{k}(x, x) \neq 1$ for all $x$.

In the last decades, the problem of merging a collection of indistinguishability operators into a single one has been addressed by different researchers (see [20, $27,28,29])$. Concretely, in [27] the functions that aggregate indistinguishability operators have been characterized by means of triangle triplets. Recently, other characterizations of those functions that aggregate indistinguishability operators have been given by Mayor and Recasens in [23], in terms of $T$-triangular triplets. Also, there are other characterizations, in terms of $T$ triangular triplets, of those functions that aggregate, the so-called relaxed indistinguishability operators in the sense of [14] (i.e., fuzzy relations verifying only properties (ii) and (iii) of a $T$-indistinguishability operator) have also been given in [7].

The rest of this contribution is organized as follows. Section 2 is devoted to the characterization of functions that merge a collection of partial $T_{i}$ indistinguishability operators into another one. In the case that all $T_{i}$ are equal to $T$, the main characterization is based on functions $F:[0,1]^{n} \rightarrow[0,1]$ that transform an $n$-dimensional $\left(T, T_{M}\right)$-tuple into a $1-$ dimensional $\left(T, T_{M}\right)$-tuple. The former characterization on $F$ is equivalent to the characterization of functions $G:[0,+\infty]^{n} \rightarrow[0,+\infty]$ that transform an $n^{-}$ dimensional $(+, \max )$-tuple into a 1 -dimensional $(+$, $\max$ )-tuple. In Subsection 2.1, we provide that a generalized inter-exchange composition property is a sufficient condition to guarantee that a function aggregates partial $T_{i}$-indistinguishability operators. Some examples of this type of functions are also provided.

\section{Aggregation of partial T-indistinguishability operators}

The problem of how to combine a collection of partial $T$-indistinguishability operators into a single one has already been addressed by Calvo el al. in [8]. They characterized the functions $F:[0,1]^{n} \rightarrow$ $[0,1]$ that merge partial $T$-indistinguishability operators into a new one by means of those functions that transform $n$-dimensional $\left(T, T_{M}\right)$-tuples into a 1dimensional $\left(T, T_{M}\right)$-tuple, where $T_{M}$ is the minimum t-norm. We recall the general idea of the above aggretation problem.

Definition 1. A function $F:[0,1]^{n} \rightarrow[0,1]$ aggregates partial $T$-indistinguishability operators if $F\left(E_{1}, \ldots, E_{n}\right)$ is a partial $T$-indistinguishability operator on $X$ for any set $X$ and any collection $\left(E_{1}, \ldots, E_{n}\right)$ of partial $T$-indistinguishability operators on $X$, where $F\left(E_{1}, \ldots, E_{n}\right)$ is the fuzzy binary relation given by $F\left(E_{1}, \ldots, E_{n}\right)(x, y)=$ $F\left(E_{1}(x, y), \ldots, E_{n}(x, y)\right)$. The funtion $F$ will be called partial indistinguishability aggregation function.

According to the previous definition and the results of the aforesaid work [8], we recall that $F=T$ aggregates partial $T$-indistinguishability operators into a new one. In particular, given a collection $\left(E_{i}\right)_{i=1}^{n}$ of partial $T_{P}$-indistinguishability operators, where $T_{P}$ is the product $t$-norm, we can ensure that the function $F\left(E_{1}, \ldots, E_{n}\right)(x, y)=\prod_{i=1}^{n} E_{i}(x, y)$ is also a partial $T_{P^{-}}$ indistinguishability operator.

Now, we recall the main characterization of the functions that merge a collection of partial $T$ indistinguishability into a single one (see [8]), since our new characterization is based on it. But, to achieve our purpose we need to recall also the next two definitions on $\left(T, T_{M}\right)$-tuples and to introduce the new type of $(+, \max )$-tuples.

Definition 2. Let $T$ be a $t$-norm. We say that $\left(a, b, c, d, d^{\prime}, d^{\prime \prime}\right) \in[0,1]^{6}$ is a (T, TM $T_{M}$-tuple if and only if $T(a, b) \leq T(c, d), T(a, c) \leq T\left(b, d^{\prime}\right), T(c, b) \leq$ $T\left(a, d^{\prime \prime}\right), a \leq \min \left\{d, d^{\prime}\right\}, b \leq \min \left\{d, d^{\prime \prime}\right\}$, and $c \leq$ $\min \left\{d^{\prime \prime}, d^{\prime}\right\}$.

The above definition can be extended to the $n$ dimentional case as follows:

Definition 3. Let $n \in \mathbb{N}$ and let $T$ be a t-norm and $\mathbf{a}, \mathbf{b}, \mathbf{c}, \mathbf{d}, \mathbf{d}^{\prime}, \mathbf{d}^{\prime \prime} \in[0,1]^{n}, n>1$, we say that $\left(\mathbf{a}, \mathbf{b}, \mathbf{c}, \mathbf{d}, \mathbf{d}^{\prime}, \mathbf{d}^{\prime \prime}\right)$ is an (n-dimensional) (T, $\left.T_{M}\right)$ tuple provided that $\left(a_{i}, b_{i}, c_{i}, d_{i}, d_{i}^{\prime}, d_{i}^{\prime \prime}\right)$ is a $\left(T, T_{M}\right)$ tuple for all $i=1, \ldots, n$, where $\mathbf{a}=\left(a_{1}, \ldots, a_{n}\right)$, $\mathbf{b}=\left(b_{1}, \ldots, b_{n}\right), \mathbf{c}=\left(c_{1}, \ldots, c_{n}\right), \mathbf{d}=\left(d_{1}, \ldots, d_{n}\right)$, $\mathbf{d}^{\prime}=\left(d_{1}^{\prime}, \ldots, d_{n}^{\prime}\right)$, and $\mathbf{d}^{\prime \prime}=\left(d_{1}^{\prime \prime}, \ldots, d_{n}^{\prime \prime}\right)$.

We mention the main characterization of the functions that merge a collection of partial $T$ indistinguishability into a single one [8].

Theorem 1. Let $n \in \mathbb{N}$ and let $F:[0,1]^{n} \rightarrow[0,1]$. The following assertions are equivalent: 
1) F aggregates partial T-indistinguishability operators,

2) $F$ transforms an $n$-dimensional (T, $\left.T_{M}\right)$-tuple into a 1-dimensional (T, $\left.T_{M}\right)$-tuple.

Now, we introduce two new concepts that we will relate to the definitions of $\left(T, T_{M}\right)$-tuples and that allows to give another characterization of those functions that aggregate partial $T$-indistinguishability operators.

Definition 4. We say that $\left(a, b, c, d, d^{\prime}, d^{\prime \prime}\right) \in$ $[0,+\infty)^{6}$ is a 1-dimensional $(+, \max )$-tuple if and only if $a+b \geq d+c, a+c \geq b+d^{\prime}, b+c \geq a+d^{\prime \prime}$, $a \geq \max \left(d, d^{\prime}\right), b \geq \max \left(d, d^{\prime \prime}\right)$, and $c \geq \max \left(d^{\prime}, d^{\prime \prime}\right)$.

The last definition can be extended to the $n$ dimensional case as follows:

Definition 5. We say that $\left(\mathbf{a}, \mathbf{b}, \mathbf{c}, \mathbf{d}, \mathbf{d}^{\prime}, \mathbf{d}^{\prime \prime}\right) \in$ $\left([0,+\infty)^{n}\right)^{6}$, where $n>1$, is an $n$-dimensional $(+, \max )$-tuple whenever $\left(a_{i}, b_{i}, c_{i}, d_{i}, d_{i}^{\prime}, d_{i}^{\prime \prime}\right)$ is a 1dimensional $(+, \max )$-tuple, for all $i=1, \ldots, n$, where $\mathbf{a}=\left(a_{1}, \ldots, a_{n}\right), \mathbf{b}=\left(b_{1}, \ldots, b_{n}\right), \mathbf{c}=\left(c_{1}, \ldots, c_{n}\right)$, $\mathbf{d}=\left(d_{1}, \ldots, d_{n}\right), \mathbf{d}^{\prime}=\left(d_{1}^{\prime}, \ldots, d_{n}^{\prime}\right)$, and $\mathbf{d}^{\prime \prime}=$ $\left(d_{1}^{\prime \prime}, \ldots, d_{n}^{\prime \prime}\right)$.

In the following theorem we deal with the two kind of tuples, i.e., $\left(T, T_{M}\right)$-tuples and $(+, \max )$-tuples.

Theorem 2. Let $T$ be a strict and continuous Archimedean t-norm with additive generator $t . \quad A \quad\left(\mathbf{a}, \mathbf{b}, \mathbf{c}, \mathbf{d}, \mathbf{d}^{\prime}, \mathbf{d}^{\prime \prime}\right)$ tuple of $\left([0,1]^{n}\right)^{6}$ is an $n$-dimensional $\left(T, T_{M}\right)$-tuple if and only if $\left(t(\mathbf{a}), t(\mathbf{b}), t(\mathbf{c}), t(\mathbf{d}), t\left(\mathbf{d}^{\prime}\right), t\left(\mathbf{d}^{\prime \prime}\right)\right)$ is an $n$-dimensional $(+, \max )$-tuple of $\left([0,+\infty)^{n}\right)^{6}$.

Proof. Calling $\mathbf{a}=\left(a_{1}, \ldots, a_{n}\right), \mathbf{b}=\left(b_{1}, \ldots, b_{n}\right)$, $\mathbf{c}=\left(c_{1}, \ldots, c_{n}\right), \mathbf{d}=\left(d_{1}, \ldots, d_{n}\right), \mathbf{d}^{\prime}=\left(d_{1}^{\prime}, \ldots, d_{n}^{\prime}\right)$, $\mathbf{d}^{\prime \prime}=\left(d_{1}^{\prime \prime}, \ldots, d_{n}^{\prime \prime}\right)$. If $\left(\mathbf{a}, \mathbf{b}, \mathbf{c}, \mathbf{d}, \mathbf{d}^{\prime}, \mathbf{d}^{\prime \prime}\right)$ is an $n^{-}$ dimensional $\left(T, T_{M}\right)$-tuple of $\left([0,1]^{n}\right)^{6}$ we have that every $\left(a_{i}, b_{i}, c_{i}, d_{i}, d_{i}^{\prime}, d_{i}^{\prime \prime}\right)$, for all $i=1, \ldots, n$ is a $\left(T, T_{M}\right)$ tuple, then

$T\left(a_{i}, b_{i}\right) \leq T\left(c_{i}, d_{i}\right) \Leftrightarrow t\left(a_{i}\right)+t\left(b_{i}\right) \geq t\left(c_{i}\right)+t\left(d_{i}\right)$,

$T\left(a_{i}, c_{i}\right) \leq T\left(b_{i}, d_{i}^{\prime}\right) \Leftrightarrow t\left(a_{i}\right)+t\left(c_{i}\right) \geq t\left(b_{i}\right)+t\left(d_{i}^{\prime}\right)$,

$T\left(c_{i}, b_{i}\right) \leq T\left(a_{i}, d_{i}^{\prime \prime}\right) \Leftrightarrow t\left(c_{i}\right)+t\left(b_{i}\right) \geq t\left(a_{i}\right)+t\left(d_{i}^{\prime \prime}\right)$,

and $a_{i} \leq \min \left(d_{i}, d_{i}^{\prime}\right), \quad b_{i} \leq \min \left(d_{i}, d_{i}^{\prime \prime}\right), \quad c_{i} \leq$ $\min \left(d_{i}^{\prime}, d_{i}^{\prime \prime}\right)$ or equivalently $t\left(a_{i}\right) \geq \max \left(t\left(d_{i}\right), t\left(d_{i}^{\prime}\right)\right)$, $b_{i} \geq \max \left(t\left(d_{i}\right), t\left(d_{i}^{\prime \prime}\right)\right), c_{i} \geq \max \left(t\left(d_{i}^{\prime}\right), t\left(d_{i}^{\prime \prime}\right)\right)$.

Furthermore, $\left(t\left(a_{i}\right), t\left(b_{i}\right), t\left(c_{i}\right), t\left(d_{i}\right), t\left(d_{i}^{\prime}\right), t\left(d_{i}^{\prime \prime}\right)\right)$ is a 1-dimensional $(+, \max )$-tuple for all $i=1, \ldots, n$ and therefore $\left(t(\mathbf{a}), t(\mathbf{b}), t(\mathbf{c}), t(\mathbf{d}), t\left(\mathbf{d}^{\prime}\right), t\left(\mathbf{d}^{\prime \prime}\right)\right)$ is an $n$-dimensional (+, max)-tuple, where $t(\mathbf{x})=$ $\left(t\left(x_{1}\right), \ldots, t\left(x_{n}\right)\right)$, for all $\mathbf{x} \in[0,1]^{n}$.

By means of the Theorems 1 and 2 we have the following characterization.
Theorem 3. Let $T$ be a strict and continuous Archimedean t-norm. A function $F:[0,1]^{n} \rightarrow[0,1]$ aggregates partial $T$-indistinguishability operators if and only if there is a function $G:[0,+\infty)^{n} \rightarrow[0,+\infty)$ defined by $G=t \circ F \circ t^{-1}$ that transforms an $n$-dimensional (+, $\max )$-tuple into a 1-dimensional $(+, \max )$-tuple, where $t$ is the additive generator of the $t-n o r m$.

Proof. $(\Rightarrow)$ First, we assume that $F$ aggregates partial $T$-indistinguishability operators which is equivalent to say that $F$ transforms an $n$-dimensional $\left(T, T_{M}\right)$ tuple into a 1 -dimensional $\left(T, T_{M}\right)$-tuple. Now, we will consider a set $X=\{x, y, z\}$ where $x, y, z$ are different elements and a collection $\left(E_{i}\right)_{i=1}^{n}$ of partial $T$-indistinguishability operators on $X$. Then, the tuple $\left(\mathbf{a}, \mathbf{b}, \mathbf{c}, \mathbf{d}, \mathbf{d}^{\prime}, \mathbf{d}^{\prime \prime}\right)$ is a $\left(T, T_{M}\right)$ tuple, where $a_{i}=E_{i}(x, y)=E_{i}(y, x), b_{i}=E_{i}(x, z)=E_{i}(z, x)$, $c_{i}=E_{i}(z, y)=E_{i}(y, z), d_{i}=E_{i}(x, x), d_{i}^{\prime}=$ $E_{i}(y, y), d_{i}^{\prime \prime}=E_{i}(z, z)$, for all $i=1, \ldots, n$. Hence, taking into account the Theorem 1 we have that $\left(F(\mathbf{a}), F(\mathbf{b}), F(\mathbf{c}), F(\mathbf{d}), F\left(\mathbf{d}^{\prime}\right), F\left(\mathbf{d}^{\prime \prime}\right)\right)$ is a $1-$ dimensional $\left(T, T_{M}\right)$-tuple, so that

$$
\begin{aligned}
& T(F(\mathbf{a}), F(\mathbf{b})) \leq T(F(\mathbf{c}), F(\mathbf{d})), \\
& T(F(\mathbf{a}), F(\mathbf{c})) \leq T\left(F(\mathbf{b}), F\left(\mathbf{d}^{\prime}\right)\right), \\
& T(F(\mathbf{c}), F(\mathbf{b})) \leq T\left(F(\mathbf{a}), F\left(\mathbf{d}^{\prime \prime}\right)\right),
\end{aligned}
$$

and

$$
\begin{gathered}
F(\mathbf{a}) \leq T_{M}\left(F(\mathbf{d}), F\left(\mathbf{d}^{\prime}\right)\right), F(\mathbf{b}) \leq T_{M}\left(F(\mathbf{d}), F\left(\mathbf{d}^{\prime \prime}\right)\right), \\
F(\mathbf{c}) \leq T_{M}\left(F\left(\mathbf{d}^{\prime}\right), F\left(\mathbf{d}^{\prime \prime}\right)\right) .
\end{gathered}
$$

From the representation theorem of the t-norm $T[22]$ the above inequalities are equivalent to the following ones

$$
\begin{aligned}
t(F(\mathbf{a}))+t(F(\mathbf{b})) & \geq t(F(\mathbf{c}))+t(F(\mathbf{d})), \\
t(F(\mathbf{a}))+t(F(\mathbf{c})) & \geq t(F(\mathbf{b}))+t\left(F\left(\mathbf{d}^{\prime}\right)\right), \\
t(F(\mathbf{c}))+t(F(\mathbf{b})) & \geq t(F(\mathbf{a}))+t\left(F\left(\mathbf{d}^{\prime \prime}\right)\right),
\end{aligned}
$$

and

$$
\begin{aligned}
& F\left(t^{-1}(t(\mathbf{a}))\right) \leq T_{M}\left(F\left(t^{-1}(t(\mathbf{d}))\right), F\left(t^{-1}\left(t\left(\mathbf{d}^{\prime}\right)\right)\right)\right), \\
& F\left(t^{-1}(t(\mathbf{b}))\right) \leq T_{M}\left(F\left(t^{-1}(t(\mathbf{d}))\right), F\left(t^{-1}\left(t\left(\mathbf{d}^{\prime \prime}\right)\right)\right)\right), \\
& F\left(t^{-1}(t(\mathbf{c}))\right) \leq T_{M}\left(F\left(t^{-1}\left(t\left(\mathbf{d}^{\prime}\right)\right)\right), F\left(t^{-1}\left(t\left(\mathbf{d}^{\prime \prime}\right)\right)\right)\right) .
\end{aligned}
$$

Now, considering in the first group of inequalities the following identity $t(F(\mathbf{x}))=\left(t \circ F \circ t^{-1}\right) t(\mathbf{x})$ for all $\mathbf{x} \in[0,1]^{n}$ and calling $G=t \circ F \circ t^{-1}$ we have that

$$
\begin{aligned}
& G(t(\mathbf{a}))+G(t(\mathbf{b})) \geq G(t(\mathbf{c}))+G(t(\mathbf{d})), \\
& G(t(\mathbf{a}))+G(t(\mathbf{c})) \geq G(t(\mathbf{b}))+G\left(t\left(\mathbf{d}^{\prime}\right)\right), \\
& G(t(\mathbf{c}))+G(t(\mathbf{b})) \geq G(t(\mathbf{a}))+G\left(t\left(\mathbf{d}^{\prime \prime}\right)\right),
\end{aligned}
$$


and since $t$ is a non-increasing function the second group of inequalities is equivalent to the following

$$
\begin{gathered}
G(t(\mathbf{a})) \geq \max \left(G(t(\mathbf{d})), G\left(t\left(\mathbf{d}^{\prime}\right)\right)\right), \\
G(t(\mathbf{b})) \geq \max \left(G(t(\mathbf{d})), G\left(t\left(\mathbf{d}^{\prime \prime}\right)\right)\right), \\
G(t(\mathbf{c})) \geq \max \left(\left(G\left(t\left(\mathbf{d}^{\prime}\right)\right), G\left(t\left(\mathbf{d}^{\prime \prime}\right)\right)\right),\right.
\end{gathered}
$$

with $\left(t(\mathbf{a}), t(\mathbf{b}), t(\mathbf{c}), t(\mathbf{d}), t\left(\mathbf{d}^{\prime}\right), t\left(\mathbf{d}^{\prime \prime}\right)\right) \in[0,+\infty)^{n}$ and where $t(\mathbf{x})=\left(t\left(x_{1}\right), \ldots, t\left(x_{n}\right)\right) \in[0,+\infty)^{n}$ for any $\mathbf{x}=\left(x_{1}, \ldots, x_{n}\right) \in[0,1]^{n}$, which shows us that $G$ transforms an $n$-dimensional $(+, \max )$-tuple into a $1-$ dimensional $(+, \max )$-tuple.

$(\Leftarrow)$ We have to see that $F$ transforms an $n$-dimensional $\left(T, T_{M}\right)$-tuple into a 1-dimensional $\left(T, T_{M}\right)$-tuple, since this is equivalent to say that $F$ aggregates partial $T$-indistinguishability operators into a new one. So that, we will consider an $n$-dimensional $\left(T, T_{M}\right)$-tuple, i.e., $\left(\mathbf{a}, \mathbf{b}, \mathbf{c}, \mathbf{d}, \mathbf{d}^{\prime}, \mathbf{d}^{\prime \prime}\right)$, and applying the Theorem 2 we obtain that $\left(t(\mathbf{a}), t(\mathbf{b}), t(\mathbf{c}), t(\mathbf{d}), t\left(\mathbf{d}^{\prime}\right), t\left(\mathbf{d}^{\prime \prime}\right)\right)$ is an $n$-dimensional (+, max)-tuple. Therefore, as $G$ transforms an $n$-dimensional $(+, \max )$-tuple into a 1 -dimensional $(+$, max $)$-tuple, we have

$$
\begin{aligned}
& G(t(\mathbf{a}))+G(t(\mathbf{b})) \geq G(t(\mathbf{c}))+G(t(\mathbf{d})), \\
& G(t(\mathbf{a}))+G(t(\mathbf{c})) \geq G(t(\mathbf{b}))+G\left(t\left(\mathbf{d}^{\prime}\right)\right), \\
& G(t(\mathbf{c}))+G(t(\mathbf{b})) \geq G(t(\mathbf{a}))+G\left(t\left(\mathbf{d}^{\prime \prime}\right)\right),
\end{aligned}
$$

and

$$
\begin{aligned}
& G(t(\mathbf{a})) \geq \max \left(G(t(\mathbf{d})), G\left(t\left(\mathbf{d}^{\prime}\right)\right)\right), \\
& G(t(\mathbf{b})) \geq \max \left(G(t(\mathbf{d})), G\left(t\left(\mathbf{d}^{\prime}\right)\right)\right), \\
& G(t(\mathbf{c})) \geq \max \left(\left(G(t(\mathbf{d})), G\left(t\left(\mathbf{d}^{\prime}\right)\right)\right),\right.
\end{aligned}
$$

and since $G=t \circ F \circ t^{-1}$, we get easily

$$
\begin{gathered}
T(F(\mathbf{a}), F(\mathbf{b})) \leq T(F(\mathbf{c}), F(\mathbf{d})), \\
T(F(\mathbf{a}), F(\mathbf{c})) \leq T\left(F(\mathbf{b}), F\left(\mathbf{d}^{\prime}\right)\right), \\
T(F(\mathbf{c}), F(\mathbf{b})) \leq T\left(F(\mathbf{a}), F\left(\mathbf{d}^{\prime \prime}\right)\right), \\
F(\mathbf{a}) \leq T_{M}\left(F(\mathbf{d}), F\left(\mathbf{d}^{\prime}\right)\right), F(\mathbf{b}) \leq T_{M}\left(F(\mathbf{d}), F\left(\mathbf{d}^{\prime \prime}\right)\right), \\
F(\mathbf{c}) \leq T_{M}\left(F\left(\mathbf{d}^{\prime}\right), F\left(\mathbf{d}^{\prime \prime}\right)\right) .
\end{gathered}
$$

Therefore, $F$ transforms an $n$-dimensional $\left(T, T_{M}\right)$ tuple into a 1-dimensional $\left(T, T_{M}\right)$-tuple.

The next result provides a necessary condition of the functions that transforms an $n$-dimensional $(+, \max )-$ tuple into a 1-dimensional $(+, \max )$-tuple.

Proposition 1. Let $G:[0, \infty)^{n} \rightarrow[0, \infty)$ be a function. 1) If $G$ is an additive function, then $G$ transforms an $n$-dimensional $(+, \max )$-tuple into a 1 dimensional (+, max)-tuple. 2) If $G$ transforms an $n$-dimensional (+, $\max )$-tuple into a 1-dimensional (+, max)-tuple then $G$ is a non-decreasing function and $G\left(\mathbf{e}+\mathbf{e}^{\prime}\right) \geq \frac{G(\mathbf{e})+G\left(\mathbf{e}^{\prime}\right)}{2}$, (inequality of Jensen).
Proof. Let $\left(\mathbf{a}^{\prime}, \mathbf{b}^{\prime}, \mathbf{c}^{\prime}, \mathbf{e}, \mathbf{e}^{\prime}, \mathbf{e}^{\prime \prime},\right)$ be an $n$-dimensional $(+$, max $)$-tuple, then $\left(a_{i}^{\prime}, b_{i}^{\prime}, c_{i}^{\prime}, e_{i}, e_{i}^{\prime}, e_{i}^{\prime \prime}\right)$ is a $1-$ dimensional $(+, \max )$-tuple for all $i=1, \ldots, n$,

$$
\begin{aligned}
& a_{i}^{\prime}+b_{i}^{\prime} \geq c_{i}^{\prime}+e_{i}, \\
& a_{i}^{\prime}+c_{i}^{\prime} \geq b_{i}^{\prime}+e_{i}^{\prime}, \\
& b_{i}^{\prime}+c_{i}^{\prime} \geq a_{i}^{\prime}+e_{i}^{\prime \prime},
\end{aligned}
$$

and $a_{i}^{\prime} \geq \max \left(e_{i}, e_{i}^{\prime}\right), b_{i}^{\prime} \geq \max \left(e_{i}, e_{i}^{\prime \prime}\right), c_{i}^{\prime} \geq$ $\max \left(e_{i}^{\prime}, e_{i}^{\prime \prime}\right)$.

Furthermore,

$$
\begin{aligned}
& \mathbf{a}^{\prime}+\mathbf{b}^{\prime} \geq \mathbf{c}^{\prime}+\mathbf{e}, \\
& \mathbf{a}^{\prime}+\mathbf{c}^{\prime} \geq \mathbf{b}^{\prime}+\mathbf{e}^{\prime}, \\
& \mathbf{c}^{\prime}+\mathbf{b}^{\prime} \geq \mathbf{a}^{\prime}+\mathbf{e}^{\prime \prime},
\end{aligned}
$$

and $\mathbf{a}^{\prime} \geq \max \left(\mathbf{e}, \mathbf{e}^{\prime}\right), \mathbf{b}^{\prime} \geq \max \left(\mathbf{e}, \mathbf{e}^{\prime \prime}\right), \mathbf{c}^{\prime} \geq$ $\max \left(\mathbf{e}^{\prime}, \mathbf{e}^{\prime \prime}\right)$. Now, considering that $G$ is an additive function and as a consequence it is a non-decreasing function, we have

$$
\begin{aligned}
& G\left(\mathbf{a}^{\prime}\right)+G\left(\mathbf{b}^{\prime}\right) \geq G\left(\mathbf{c}^{\prime}\right)+G(\mathbf{e}), \\
& G\left(\mathbf{a}^{\prime}\right)+G\left(\mathbf{c}^{\prime}\right) \geq G\left(\mathbf{b}^{\prime}\right)+G\left(\mathbf{e}^{\prime}\right), \\
& G\left(\mathbf{c}^{\prime}\right)+G\left(\mathbf{b}^{\prime}\right) \geq G\left(\mathbf{a}^{\prime}\right)+G\left(\mathbf{e}^{\prime \prime}\right),
\end{aligned}
$$

and $G\left(\mathbf{a}^{\prime}\right) \geq \max \left(G(\mathbf{e}), G\left(\mathbf{e}^{\prime}\right)\right), \quad G\left(\mathbf{b}^{\prime}\right) \geq$ $\max \left(G(\mathbf{e}), G\left(\mathbf{e}^{\prime \prime}\right)\right), \quad G\left(\mathbf{c}^{\prime}\right) \geq \max \left(G\left(\mathbf{e}^{\prime}\right), G\left(\mathbf{e}^{\prime \prime}\right)\right)$. Therefore, $\left(G\left(\mathbf{a}^{\prime}\right), G\left(\mathbf{b}^{\prime}\right), G\left(\mathbf{c}^{\prime}\right), G(\mathbf{e}), G\left(\mathbf{e}^{\prime}\right), G\left(\mathbf{e}^{\prime \prime}\right)\right)$ is a 1-dimensional $(+, \max )$-tuple.

In the second case, we consider the following $n$-dimensional $\quad(+, \max )$-tuple $\left(\mathbf{e}+\mathbf{e}^{\prime}, \mathbf{e}+\mathbf{e}^{\prime \prime}, \mathbf{e}^{\prime}+\mathbf{e}^{\prime \prime}, \mathbf{e}, \mathbf{e}^{\prime}, \mathbf{e}^{\prime \prime}\right)$ for any $\mathbf{e}, \mathbf{e}^{\prime}, \mathbf{e}^{\prime \prime} \in$ $[0,+\infty]^{n}$. As $G$ transforms an $n$-dimensional $(+, \max )$-tuple into a 1-dimensional $(+, \max )$-tuple, we have

1) $G\left(\mathbf{e}+\mathbf{e}^{\prime}\right)+G\left(\mathbf{e}+\mathbf{e}^{\prime \prime}\right) \geq G(\mathbf{e})+G\left(\mathbf{e}^{\prime}+\mathbf{e}^{\prime \prime}\right)$

2) $G\left(\mathbf{e}+\mathbf{e}^{\prime}\right)+G\left(\mathbf{e}^{\prime}+\mathbf{e}^{\prime \prime}\right) \geq G\left(\mathbf{e}^{\prime}\right)+G\left(\mathbf{e}+\mathbf{e}^{\prime \prime}\right)$,

3) $G\left(\mathbf{e}+\mathbf{e}^{\prime \prime}\right)+G\left(\mathbf{e}^{\prime}+\mathbf{e}^{\prime \prime}\right) \geq G\left(\mathbf{e}^{\prime \prime}\right)+G\left(\mathbf{e}+\mathbf{e}^{\prime}\right)$,

and

4) $G\left(\mathbf{e}+\mathbf{e}^{\prime}\right) \geq \max \left(G(\mathbf{e}), G\left(\mathbf{e}^{\prime}\right)\right) \geq G(\mathbf{e})$,

5) $G\left(\mathbf{e}+\mathbf{e}^{\prime \prime}\right) \geq \max \left(G(\mathbf{e}), G\left(\mathbf{e}^{\prime \prime}\right)\right) \geq G(\mathbf{e})$,

6) $G\left(\mathbf{e}^{\prime}+\mathbf{e}^{\prime \prime}\right) \geq \max \left(G\left(\mathbf{e}^{\prime}\right), G\left(\mathbf{e}^{\prime \prime}\right)\right) \geq G\left(\mathbf{e}^{\prime}\right)$.

From 4) or 5) or 6 ) we have that $G$ is a non-decreasing function.

From 1) and 2) we get $2 G\left(\mathbf{e}+\mathbf{e}^{\prime}\right) \geq G(\mathbf{e})+G\left(\mathbf{e}^{\prime}\right)$,

From 1) and 3) we get $2 G\left(\mathbf{e}+\mathbf{e}^{\prime \prime}\right) \geq G(\mathbf{e})+G\left(\mathbf{e}^{\prime \prime}\right)$,

From 2) and 3) we get $2 G\left(\mathbf{e}^{\prime}+\mathbf{e}^{\prime \prime}\right) \geq G\left(\mathbf{e}^{\prime}\right)+G\left(\mathbf{e}^{\prime \prime}\right)$. Therefore, $G\left(\mathbf{e}+\mathbf{e}^{\prime}\right) \geq \frac{G(\mathbf{e})+G\left(\mathbf{e}^{\prime}\right)}{2}$. 


\subsection{Aggregation of partial indistinguishability operators defined by different t-norms}

Here, the problem is how to combine a collection $\left(E_{i}\right)_{i=1}^{n}$ of partial $T_{i}$-indistinguishability operators into a single one. To achieve this aim, we need to introduce the notion of partial $T_{i}$-indistinguishability operators aggregation function.

Definition 6. A function $F:[0,1]^{n} \rightarrow[0,1]$ aggregates a collection $\left(E_{i}\right)_{i=1}^{n}$ of partial $T_{i}$-indistinguishability operators on any non-empty set $X$ into a new partial $T$-indistinguishability operator if $F\left(E_{1}, \ldots, E_{n}\right)$ is partial T-indistinguishability operator on $X$, where $F\left(E_{1}, \ldots, E_{n}\right)$ is the fuzzy binary relation given by $F\left(E_{1}, \ldots, E_{n}\right)(x, y)=$ $F\left(E_{1}(x, y), \ldots, E_{n}(x, y)\right)$. The function $F$ should be called partial $T_{i}$-indistinguishability operators aggregation function.

Next, we give sufficient condition to guarantee that a function aggregates partial $T_{i}$-indistinguishability operators. In order to achieve it, we need to introduce the generalized version of the inter-exchange composition functions condition (ICFE) introduced in [8].

Definition 7. Let $T_{i}, i=1, \ldots, n$ be a family of $t$-norms. We will say that a $t$-norm $T$ and a function $F:[0,1]^{n} \rightarrow[0,1]$ satisfy the generalized interexchange composition functions (GICFE, for short) provided that

$$
T(F(\mathbf{a}), F(\mathbf{b}))=F\left(T_{1}\left(a_{1}, b_{1}\right), \ldots, T_{n}\left(a_{n}, b_{n}\right)\right),
$$

for all $\mathbf{a}, \mathbf{b} \in[0,1]^{n}$.

Note that, if all $T_{i}$ are equal to $T$ the above condition coincides with the inter-exchange composition functions equality (ICFE) whose condition holds by every t-norm $T$ and every $i$-th projection. We also know that there are functions that aggregate partial $T$-indistinguishability operators but they do not satisfy the ICFE (see [8]). By means of Definition 7 we can show the following.

Proposition 2. Let $E_{i}, i=1, \ldots, n$ be a family of partial $T_{i}$-indistinguishability operators, and let $T$ be another t-norm. If $F:[0,1]^{n} \rightarrow[0,1], n \geq 1$, is a non-decreasing function and $T$ satisfy the generalized inter-exchange composition functions, then $F$ aggregates the collection $\left(E_{i}\right)_{i=1}^{n}$ of partial $T_{i}$-indistinguishability operators into a partial $T$-indistinguishability operator.

Proof. We need to show that $F\left(E_{1}, \ldots, E_{n}\right)$ is a partial $T$-indistinguishability operator. The symmetry follows directly from the symmetry of each $E_{i}$. The next inequality $F\left(E_{1}, \ldots, E_{n}\right)(x, y) \leq$
$F\left(E_{1}, \ldots, E_{n}\right)(x, x)$ follows from the increasingness of $F$ and from the inequality $E_{i}(x, y) \leq E_{i}(x, x)$, for all $i=1, \ldots, n$.

Now we must to show that,

$$
\begin{gathered}
T\left(F\left(E_{1}(x, z), \ldots, E_{n}(x, z)\right), F\left(E_{1}(z, y), \ldots, E_{n}(z, y)\right)\right) \\
\leq \\
T\left(F\left(E_{1}(x, y), \ldots, E_{n}(x, y)\right), F\left(E_{1}(z, z), \ldots, E_{n}(z, z)\right)\right) .
\end{gathered}
$$

As each $E_{i}$ is a partial $T_{i}$-indistinguishability operator we have that

$$
T_{i}\left(E_{i}(x, z), E_{i}(z, y)\right) \leq T_{i}\left(E_{i}(x, y), E_{i}(z, z)\right)
$$

and since $F$ is non-decreasing we obtain

$$
\begin{gathered}
F\left(T_{1}\left(E_{1}(x, z), E_{1}(z, y)\right), \ldots, T_{n}\left(E_{n}(x, z), E_{n}(z, y)\right)\right) \\
\leq \\
F\left(T_{1}\left(E_{1}(x, y), E_{1}(z, z)\right), \ldots, T_{n}\left(E_{n}(x, y), E_{n}(z, z)\right)\right) .
\end{gathered}
$$

Applying the given condition we have

$T\left(F\left(E_{1}(x, z), \ldots, E_{n}(x, z)\right), F\left(E_{1}(z, y), \ldots, E_{n}(z, y)\right)\right)=$

$F\left(T_{1}\left(E_{1}(x, z), E_{1}(z, y)\right), \ldots, T_{n}\left(E_{n}(x, z), E_{n}(z, y)\right)\right) \leq$

$F\left(T_{1}\left(E_{1}(x, y), E_{1}(z, z)\right), \ldots, T_{n}\left(E_{n}(x, y), E_{n}(z, z)\right)\right)=$

$T\left(F\left(E_{1}(x, y), \ldots, E_{n}(x, y)\right), F\left(E_{1}(z, z), \ldots, E_{n}(z, z)\right)\right)$.

To sum up, $F\left(E_{1}, \ldots, E_{n}\right)$ is a partial $T$ indistinguishability operator, this means that $F$ aggregates a collection of partial $T_{i}$-indistinguishability operators into a partial $T$-indistinguishability operator.

The next example shows that there are functions that aggregate partial $T_{i}$-indistinguishability operators but they do not satisfy the GICFE.

Example 1. Fix $k \in] 0,1\left[\right.$. Consider the function $F_{k}$ : $[0,1]^{n} \rightarrow[0,1]$ defined by

$$
F_{k}(\mathbf{a})=k,
$$

for all $\mathbf{a} \in[0,1]^{n}$. It is not hard to verify that $F_{k}$ aggregates a collection $\left(E_{i}\right)_{i=1}^{n}$ of partial $T_{i}$-indistinguishability operators into a partial $T_{P}$-indistinguishability operator. However, $F_{k}, T_{P}$ and any $T_{i}, i=1, \ldots, n$ do not satisfy the GICFE. Indeed,

$$
\begin{gathered}
k^{2}=T_{P}(k, k)=T_{P}\left(F_{k}(\mathbf{a}), F_{k}(\mathbf{b})\right)< \\
F_{k}\left(T_{1}\left(a_{1}, b_{1}\right), \ldots, T_{n}\left(a_{n}, b_{n}\right)\right)=k,
\end{gathered}
$$

for all $\mathbf{a}, \mathbf{b} \in[0,1]^{n}$.

Taking into account the given results in [20], we can show the following 
Proposition 3. Let $F:[0,1]^{n} \rightarrow[0,1]$ be a function and $T, T_{1}, T_{2}, \ldots, T_{n}$ continuous Archimedean $t$-norms with additive generators, $t, t_{1}, \ldots, t_{n}$ respectively. If there exists an additive function $s:\left(\mathbb{R}^{+}\right)^{n} \rightarrow \mathbb{R}^{+}$such that $F=t^{(-1)} \circ s \circ\left(t_{1} \times \ldots \times t_{n}\right)$, then $F$ aggregates a collection $\left(E_{i}\right)_{i=1}^{n}$ of partial $T_{i}$-indistinguishability operators into a partial $T$-indistinguishability operator.

Proof. We need to show the three conditions of a partial $T$-indistinguishability operator.

To see the first condition of a partial $T$-indistinguishability operator, i.e., $F\left(E_{1}, \ldots, E_{n}\right)(x, y) \leq F\left(E_{1}, \ldots, E_{n}\right)(x, x)$, we need to distinguish several cases, in all of them we know that each $E_{i}$ satisfies $E_{i}(x, y) \leq E_{i}(x, x)$ and $s \circ\left(t_{1} \times \ldots \times t_{n}\right)$ is a non-decreasing function.

1. If $s\left(\left(t_{1}\left(E_{1}(x, y)\right), \ldots, t_{n}\left(E_{n}(x, y)\right)\right)\right) \leq t(0)$ and $s\left(\left(t_{1}\left(E_{1}(x, x)\right), \ldots, t_{n}\left(E_{n}(x, x)\right)\right)\right) \leq t(0)$, due to the above considerations and from the fact that $t^{(-1)}=t^{-1}$ we get

$$
\begin{array}{ll}
F\left(E_{1}, \ldots, E_{n}\right)(x, y) & = \\
t^{-1} \circ s \circ\left(t_{1} \times \ldots \times t_{n}\right)\left(E_{1}, \ldots, E_{n}\right)(x, y) & \leq \\
t^{-1} \circ s \circ\left(t_{1} \times \ldots \times t_{n}\right)\left(E_{1}, \ldots, E_{n}\right)(x, x) & = \\
F\left(E_{1}, \ldots, E_{n}\right)(x, x) &
\end{array}
$$

2. If $s\left(\left(t_{1}\left(E_{1}(x, y)\right), \ldots, t_{n}\left(E_{n}(x, y)\right)\right)\right)>t(0)$ the inequality is also true.

3. If $s\left(\left(t_{1}\left(E_{1}(x, x)\right), \ldots, t_{n}\left(E_{n}(x, x)\right)\right)\right)>t(0)$, as we know that

$$
\begin{aligned}
& t(0)<s\left(\left(t_{1}\left(E_{1}(x, x)\right), \ldots, t_{n}\left(E_{n}((x, x))\right)\right) \leq\right. \\
& s\left(\left(t_{1}\left(E_{1}(x, y), \ldots, t_{n}\left(E_{n}(x, y)\right)\right)\right)\right.
\end{aligned}
$$

then the inequality follows directly.

Now, we will show the third condition of a partial $T$-indistinguishability operator to achieve that $F\left(E_{1}, \ldots, E_{n}\right)$ is a partial $T$-indistinguishability operator, i.e.,

$$
\begin{gathered}
T\left(F\left(E_{1}, \ldots, E_{n}\right)(x, z), F\left(E_{1}, \ldots, E_{n}\right)(z, y)\right) \\
\leq \\
T\left(F\left(E_{1}, \ldots, E_{n}\right)(x, y), F\left(E_{1}, \ldots, E_{n}\right)(z, z)\right),
\end{gathered}
$$

for this we will consider the representation theorem of the t-norm $T$ [22]

To guarantee the previous inequality we need to split the proof in two cases:

1. If $T$ is a strict t-norm and $E_{i}, i=1, \ldots, n$ are partial $T_{i}$-indistinguishability operators we know that

$$
T_{i}\left(E_{i}(x, z), E_{i}(z, y)\right) \leq T_{i}\left(E_{i}(x, y), E_{i}(z, z)\right)
$$

or equivalently

$$
\left.t_{i}\left(E_{i}(x, z)\right)+t_{i}\left(E_{i}(z, y)\right) \geq t_{i}\left(E_{i}(x, y)\right)+E_{i}(z, z)\right) .
$$

Considering this inequality, the following notation $\mathbf{a}=\left(t_{1}\left(E_{1}(x, z)\right), \ldots, t_{n}\left(E_{n}(x, z)\right)\right)$, $\mathbf{b}=\left(t_{1}\left(E_{1}(z, y)\right), \ldots, t_{n}\left(E_{n}(z, y)\right)\right)$, $\mathbf{c}=\left(t_{1}\left(E_{1}(x, y)\right), \ldots, t_{n}\left(E_{n}(x, y)\right)\right)$ and $\mathbf{d}=\left(t_{1}\left(E_{1}(z, z)\right), \ldots, t_{n}\left(E_{n}(z, z)\right)\right)$, and the properties of $s$ and $t^{-1}$ functions, we obtain

$$
t^{-1}(s(\mathbf{a})+s(\mathbf{b})) \leq t^{-1}(s(\mathbf{c})+s(\mathbf{d})) .
$$

Now, replacing the function $s$ by $s=i d \circ s=$ $t \circ t^{-1} \circ s$, and considering the representation theorem of a t-norm [22], we get the following equivalent inequality

$$
\begin{gathered}
T\left(\left(t^{-1} \circ s\right)(\mathbf{a}),\left(t^{-1} \circ s\right)(\mathbf{b})\right) \leq \\
T\left(\left(t^{-1} \circ s\right)(\mathbf{c}),\left(t^{-1} \circ s\right)(\mathbf{d})\right) .
\end{gathered}
$$

2. If $T$ is a non-strict t-norm we split the proof as follows

(a) If $s\left(t_{1}\left(E_{1}(x, z)\right), \ldots, t_{n}\left(E_{n}(x, z)\right)\right)>t(0)$ or $s\left(t_{1}\left(E_{1}(z, y)\right), \ldots, t_{n}\left(E_{n}(z, y)\right)\right)>t(0)$, then the condition (iii) is true.

(b) If $s\left(t_{1}\left(E_{1}(x, z)\right), \ldots, t_{n}\left(E_{n}(x, z)\right)\right) \leq t(0)$ and $s\left(t_{1}\left(E_{1}(z, y)\right), \ldots, t_{n}\left(E_{n}(z, y)\right)\right) \leq t(0)$, as $t^{(-1)}=t^{-1}$ the proof follows in the same way of the strict t-norm.

Furthermore, $F\left(E_{1}, \ldots, E_{n}\right)$ also satisfies the condition (iii) of a partial $T$-indistinguishability operator and therefore $F\left(E_{1}, \ldots, E_{n}\right)$ is a partial $T$-indistinguishability operator.

The function $F$ of the Proposition 3 satisfies the condition of the Definition 7 as we see in the next result.

Proposition 4. The function $F=t^{-1} \circ s \circ\left(t_{1} \times\right.$ $\left.\ldots \times t_{n}\right)$, where $t, t_{1}, \ldots, t_{n}$ are additive generators of a collection of strict continuous Archimedean t-norms $T, T_{1}, T_{2}, \ldots, T_{n}$, and $s:\left(\mathbb{R}^{+}\right)^{n} \rightarrow \mathbb{R}^{+}$is an additive function, the following condition holds

$$
T(F(\mathbf{a})), F(\mathbf{b}))=F\left(T_{1}\left(a_{1}, b_{1}\right), \ldots, T_{n}\left(a_{n}, b_{n}\right)\right) .
$$

Proof. This is a matter of a simple computation.

Observe that, in the Proposition 3 we can consider that all operators $E_{i}, i=1, \ldots, n$, are partial indistinguishability operators w.r.t the same t-norm $T$, with additive generator $t$. Then, the function $F$ should be $F=t^{(-1)} \circ s \circ(t \times \ldots \times t)$. 
Remark 1. Note that, any function $F=t^{-1} \circ s \circ(t \times$ $\ldots \times t)$, where $t$ is the additive generator of any strict $t$-norm $T$ and $s:\left(\mathbb{R}^{+}\right)^{n} \rightarrow \mathbb{R}^{+}$is an additive function, satisfies the condition ICFE given in [8] which guarantees that the function $F$ aggregates a collection of partial T-indistinguishability operators.

Example 2. Considering in the above expression of $F=t^{-1} \circ s \circ(t \times \ldots \times t)$ the function $s\left(x_{1}, \ldots, x_{n}\right)=$ $\sum_{i=1}^{n} w_{i} x_{i}$, with $\sum_{i=1}^{n} w_{i}=1$, and taking the well-known additive generators $t$ of different $t$-norms, we have

1. If $t(x)=-\ln x$, generator of the product $t$-norm, then $F\left(x_{1}, \ldots, x_{n}\right)=\prod_{i=1}^{n} x_{i}^{w_{i}}$. This function $F$ is a weighted geometric mean.

2. If $t(x)=\frac{1-x^{\lambda}}{\lambda}$ with $\left.\lambda \in\right]-\infty, 0[$, the generator of a Schweizer-Sklar t-norm, then $F\left(x_{1}, \ldots, x_{n}\right)=\left(1-\sum_{i=1}^{n} w_{i}\left(1-x_{i}^{\lambda}\right)\right)^{1 / \lambda}$. This is a weighted power mean whose generator is $g(x)=1-x^{\lambda}$.

3. If $t(x)=(-\ln x)^{\lambda}$ with $\left.\lambda \in\right] 0,+\infty[$, generator of a Aczél-Alsina t-norm, then

$F\left(x_{1}, \ldots, x_{n}\right)=e^{-\left(\sum_{i=1}^{n} w_{i}\left(-\ln x_{i}\right)^{\lambda}\right)^{1 / \lambda}}$. This is a weighted quasi-arithmetic mean whose generator is $g(x)=(-\ln x)^{\lambda}$.

Now, we mention a specific class of non-decreasing weighted functions that fit with the aggregation process of this work.

Proposition 5. The function

$$
F\left(a_{1}, \ldots, a_{n}\right)=t^{-1}\left(\sum_{i=1}^{n} w_{i} t_{i}\left(a_{i}\right)\right)=t^{-1} \circ M_{\mathbf{w}}\left(t_{i}\left(a_{i}\right)\right)
$$

with $M_{\mathbf{w}}$ denoting the weighted mean, where $w_{i}>0$, for all $i=1, \ldots, n$ and $\sum_{i=1}^{n} w_{i}=1$, and $t, t_{i}$ are additive generators of the given srict continuous Archimedean t-norms $T, T_{i}$, respectively, aggregates a collection $\left(E_{i}\right)_{i=1}^{n}$ of partial $T_{i}$-indistinguishability operators into another partial $T$-indistinguishability operator.

Proof. The (i) and (ii) conditions of any partial $T$ indistinguishability operator follow directly from the same conditions of the $E_{i}, i=1, \ldots, n$ partial $T_{i}^{-}$ indistinguishability operators and from the increasingness of $F$. From now on, we will show the condition (iii) of a partial $T$-indistinguishability operator. Because of any $E_{i}, i=1, \ldots, n$, is a partial $T_{i}^{-}$ indistinguishability operator, we know that

$$
T_{i}\left(E_{i}(x, z), E_{i}(z, y)\right) \leq T_{i}\left(E_{i}(x, y), E_{i}(z, z)\right)
$$

and taking into account the representation theorem of the $\mathrm{t}$-norm [22], we have that

$$
t_{i}\left(E_{i}(x, z)\right)+t_{i}\left(E_{i}(z, y)\right) \geq t_{i}\left(E_{i}(x, y)\right)+t_{i}\left(E_{i}(z, z)\right)
$$

and from this

$$
\begin{aligned}
& t^{-1}\left(\sum_{i=1}^{n} w_{i} t_{i}\left(E_{i}(x, z)\right)+\sum_{i=1}^{n} w_{i} t_{i}\left(E_{i}(z, y)\right)\right) \leq \\
& t^{-1}\left(\sum_{i=1}^{n} w_{i} t_{i}\left(E_{i}(x, y)\right)+\sum_{i=1}^{n} w_{i} t_{i}\left(E_{i}(z, z)\right)\right)
\end{aligned}
$$

and considering that $I_{d}=t \circ t^{-1}$ in the above sumation we get the following equivalent expression

$T\left(F\left(E_{1}, \ldots, E_{n}\right)(x, z), F\left(E_{1}, \ldots, E_{n}\right)(z, y)\right)$

$=t^{-1}\left(t\left(F\left(E_{1}, \ldots, E_{n}\right)(x, z)\right), t\left(F\left(E_{1}, \ldots, E_{n}\right)(z, y)\right)\right)$

$\leq t^{-1}\left(t\left(F\left(E_{1}, \ldots, E_{n}\right)(x, y)\right), t\left(F\left(E_{1}, \ldots, E_{n}\right)(z, z)\right)\right)$

$=T\left(F\left(E_{1}, \ldots, E_{n}\right)(x, y), F\left(E_{1}, \ldots, E_{n}\right)(z, z)\right)$,

furthermore $F\left(E_{1}, \ldots, E_{n}\right)$ satisfies the condition (iii) of a partial $T$-indistinguishability operator. Therefore, $F\left(E_{1}, \ldots, E_{n}\right)$ is a partial $T$-indistinguishability operator.

\section{Acknowledgement}

This work is partially supported by Ministry of Economy and Competitiveness under contract DPI201786372-C3-3-R (AEI, FEDER, UE), and by the Vicerectorate for Research and Transference, UAH.

\section{References}

[1] B. De Baets and R. Mesiar, Pseudo-metrics and T-equivalences, J. Fuzzy Math., 5 (1997), 471481.

[2] B. De Baets and R. Mesiar, Metrics and Tequalities, J. Math. Anal. Appl., 267 (2002), 531547.

[3] G. Beliakov, H. Bustince and T. Calvo-Sánchez, A Practical Guide to Averaging Functions, Springer, Heidelberg, Berlin, New York, 2016.

[4] G. Beliakov, A. Pradera and T. Calvo, Aggregation Functions: a guide for practitioners, Springer, Heidelberg, Berlin, New York, 2007.

[5] B. Bouchon-Meunier, (Ed.), Aggregation and Fusion of Imperfect Information, Sudies in Fuzziness and Soft Computing, Vol. 12, Physica-Verlag, Heidelberg, 1998.

[6] M. Bukatin, R. Kopperman and S.G. Matthews, Some corollaries of the correspondence between partial metric and multivalued equalities, Fuzzy Set. Syst. 256 (2014), 57-72. 
[7] T. Calvo, P. Fuster-Parra and O. Valero, On the problem of relaxed indistinguishability aggregation operators, Proc. of the Workshop on Applied Topological Structures, WATS'17, (2017), 19-26.

[8] T. Calvo, P. Fuster-Parra and O. Valero, Aggregation on partial indistinguishability aggregation operators, Proc. 17th International Conference on Information Processing and Management of Uncertainty in Knowledge-Based Systems, IPMU'18, Vol. I, (2018), 207-218.

[9] M. Demirci, The order-theoretic duality and relations between partial metrics and local equalities, Fuzzy Set. Syst. 192 (2011) 45-57.

[10] M.M. Deza and E. Deza, Encyclopedia of Distances, Springer-Verlag, 2016.

[11] J. Drewniak and U. Dudziak, Aggregation preserving classes of fuzzy relations, Kybernetica, 41 (3), (2005), 265-284.

[12] U. Dudziak, Preservation of $t$-norm and $t-$ conorm based properties of fuzzy relations during aggregation process, Proc. 8th Conference of European Society for Fuzzy Logic and Technology, EUSFLAT'2013, (2013), 376-383.

[13] P. Fuster-Parra, J. Martín, J. J. Miñana and O. Valero, A study on the relationship between relaxed metrics and indistinguishability operators, 2017, (submitted).

[14] P. Fuster-Parra, J. Martín, J. Recasens and O. Valero, On the metric behavior of partial indistinguishability operators, preprint.

[15] S. Gottwald, On t-norms which are related to distances of fuzzy sets, BUSEFAL 50 (1992), 25-30.

[16] M. Grabisch, J.L. Marichal, R. Mesiar and E. Pap, Aggregation Functions, Cambridge University Press, New York, 2009.

[17] P. Klement, R. Mesiar and E. Pap, Triangular norms, Kluwer, Dordrecht, 2000.

[18] O. Grigorenko and J. Lebendinska, On another view of aggregation of fuzzy relations, In: S. Galichet, J. Montero and G. Mauris, (eds), Proc. 7th. Conf. EUSFLAT'2011 and LFA'2011, Atlantis Press, (2011), 21-27.

[19] U. Höhle, Fuzzy equalities and indistinguishability, Proc. of EUFIT'93, Vol. 1, Aachen, 1993 pp. 358-363.

[20] J. Jacas and J. Recasens, Aggregation of TTransitive Relations, Int. J. Intell. Syst. 18 (2003), 1193-1214.
[21] P. Klement, R. Mesiar and E. Pap, Triangular Norms, Kluwer, Dordrecht, 2000.

[22] C. M. Ling, Representation of associative function, Publ. Math. Debrecen 12, (1965), 189-212.

[23] G. Mayor and J. Recasens, Preserving Ttransitivity in: Artificial Intelligence Research and Development, A. Nebot et al. (eds), IOS Press, Vol. 288, 79-87, 2016.

[24] S. Ovchinnikov, Representation of transitive fuzzy relations, Aspects of Vageness (H. Skala, S. Termini and E. Trillas, eds.), Reidel, Dordrecht, 1984, pp. 105-118.

[25] S. Ovchinnikov, Similarity relations, fuzzy partitions, and fuzzy orderings, Fuzzy Sets and Syst., 40 (1991), 107-126.

[26] V. Peneva and I. Popchev, Properties of the aggregation operators related with fuzy relations, Fuzzy Sets and Syst., 139 (3), (2003), 615-633.

[27] A. Pradera, E. Trillas, and E. Castiñeira. On distances aggregation, in: Proc. of Information Processing and Management of Uncertainty in Knowledge-Based Systems International Conference, B. Bouchon-Meunier et al. (eds), Universidad Politécnica de Madrid Press, Vol. II, 693-700, 2000.

[28] A. Pradera, E. Trillas and E. Castiñeira, On the aggregation of some classes of fuzzy relations, in: Technologies for constructing intelligent systems, B. Bouchon-Meunier, J. Gutierrez, L. Magdalena and R. Yager (eds), Springer-Verlag, 125-147, 2002 .

[29] J. Recasens, Indistinguishability Operators: Modelling Fuzzy Equalities and Fuzzy Indistinguishability Relations, Springer, Berlin, 2010 .

[30] E. Trillas, Assaig sobre les relacions d'indistingibilitat, Proc. Primer Congrés Catalá de Lógica Matemática, Barcelona, 1982, pp. $51-59$.

[31] L. Valverde, On the structure of Findistinguishability operators, Fuzzy Set. Syst. 17 (1985) 313-328.

[32] L. A. Zadeh, Similarity relations and fuzzy orderings, Inform. Sci. 3(2), (1971), 177-200. 